\title{
External Cephalic Version Success Rate and Associated Factors: Experience from a Tertiary Center in Sub-Saharan Africa: A Cross-sectional Study
}

Abraham Fessehaye ( $\nabla$ abrahamfessehaye4@gmail.com )

St. Paul's Hospital Millennium Medical College

Wondimu Gudu

St. Paul's Hospital Millennium Medical College

Tadesse Urgie

St. Paul's Hospital Millennium Medical College

Gebeyehu Masresha

St. Paul's Hospital Millennium Medical College

\section{Research Article}

Keywords: External Cephalic Version Success rate, associated factors, Tertiary Center , Sub-Saharan Africa

Posted Date: September 29th, 2021

DOl: https://doi.org/10.21203/rs.3.rs-869607/v1

License: (c) (i) This work is licensed under a Creative Commons Attribution 4.0 International License. Read Full License 


\section{Abstract}

Background: External cephalic version (ECV), which is now routinely offered in developed countries, is not a popular procedure in the developing regions, such as Sub-Saharan Africa (9). There is also scarce data on success rate of ECV from this developing region of Africa. The aim of this study was to determine the success rate of ECV and it's associated factors in an Ethiopian setting.

Methods: A total of 152 ECVs performed at the St. Paul's Hospital Millennium Medical College, Addis Ababa, Ethiopia from June 1, 2018 up to March 30, 2019 were analyzed, using a cross-sectional study design. Patients with breech presentation were recruited at 36 weeks of gestation or more during antenatal consultations and ECV procedures were provided by Maternal-Fetal-Medicine (MFM) specialist or MFM fellow at Ultrasound room. Data was analyzed using SPSS version 21. Chi-square test of association was done for categorical data analysis. Multivariate logistic regression analysis was used to determine predictors of success of ECV. Odds ratio, $95 \% \mathrm{Cl}$, and P-value were used to describe findings' significance

Results: The success rate of ECV was 71.7\%. Multiparous women had a 1.4-times higher probability of success than nulliparous women $(95 \% \mathrm{Cl}, 0.07-2.35)$; thin abdominal wall had 3.5 fold increased success rate ( $95 \% \mathrm{Cl}, 0.29-42.40)$; unengaged breech had a 1.1 higher probably of success than engaged $(95 \% \mathrm{Cl}$, 0.26-4.74); a posterior placental location increased the success rate by 1.14 times compared with an anterior placenta (95\% Cl, 1.03-2.60); the odds of successful ECV was $14.68((95 \% \mathrm{Cl}, 1.65-34.97)$ when cases experienced no pain during the procedure ; and tense uterine tone was associated a lower success rate as compared to soft tone ( $\mathrm{AOR}=0.08,95 \% \mathrm{Cl}, 0.016-0.39$ ). Eighty-four percent of those mothers who had successful ECV had spontaneous vertex vaginal delivery.

Conclusion: The success rate of ECV in this study is found to be $71.7 \%$, which is higher than reports from previous studies. Multiparty, absence of pain during procedure, posterior placenta, unengaged breech, soft uterine tone, and thin abdominal wall were significantly associated with ECV success.

Précis: The success rate of ECV in this study is found to be $71.7 \%$, which is higher than reports from previous studies.

\section{Introduction}

Breech presentation complicates $3-4 \%$ of all pregnancies at term and many of these pregnancies are delivered by caesarean Sect. $(1,2)$. External cephalic version (ECV) is an obstetric procedure that aims to rotate a breech fetus into the cephalic position by external maneuver through the maternal abdomen(3).

ECV decreases the likelihood that the fetus will be in a non-cephalic presentation at birth(4-6). With a number needed to treat of three, it still remains a very efficient procedure to prevent a cesarean delivery (7). There is not enough evidence from randomized trials to assess complications of ECV at term but large observational studies suggest that complications are rare (8). 
External cephalic version (ECV), which is now routinely offered in developed countries, is not a popular procedure in the developing regions, such as Sub-Saharan Africa (9). There is also scarce data on success rate of ECV from this developing region of Africa. The aim of this study was to determine the success rate of ECV and it's associated factors in an Ethiopian setting.

\section{Methods}

Data for 152 ECV cases that were performed at the St.Paul's Hospital Millennium Medical College (Addis Ababa, Ethiopia) from June1, 2018 to March 30, 2019 were collected prospectively. The study was conducted using a cross-sectional study design. Mothers with singleton pregnancy in breech presentation were recruited at $\geq 36$ weeks of gestation during antenatal consultations. ECV procedures were provided by Maternal-Fetal-Medicine (MFM) specialist or MFM fellow at ultrasound room. Exclusion criteria were the presence of placenta previa, placental abruption, oligohydramnios, fetal compromise, fetal death, severe fetal malformation, multiple gestation, Rhesus sensitization, uterine abnormalities, and clotting disorders.

On the day of the scheduled ECV, eligible participants had an ultrasound scan with the goals of estimating fetal weight, determining type of breech; placental location; and amount of amniotic fluid. ECV was performed one hour after administration of a single dose oral administration of $20 \mathrm{mg}$ Nifedipin. The ECV procedure was carried out under ultrasound assessment, with the mother in a slight Trendelenburg position, and a ready $\mathrm{C} / \mathrm{S}$ operating theatre for emergency interventions. Fetal heart rate monitoring was used to assess fetal well-being before and after the procedure. A change in presentation from podalic to cephalic was considered a successful ECV.

Data on maternal age, maternal weight, gestational age, parity, amount of amniotic fluid, placental location, and type of breech were collected on the same day immediately before performing ECV. Sonographic and procedural variables were collected during the procedure and immediately after the procedure. Mode of delivery was reviewed from maternal charts. Data was collected by midwives and the principal investigator regularly supervised data consistency and completion.

Data was entered into and analyzed using SPSS version 21. Chi-square test of association was done for categorical data analysis. Bivariate and multivariate logistic regression analysis was used to determine predictors of success of ECV. Odds ratio, $95 \% \mathrm{Cl}$, and P-value were used to describe findings' significance.

\section{Results}

A total of 152 case of ECV were recruited in this study. The overall success rate of ECV during the study period was $71.7 \%$ (109/152). There was no difference in socio-demographic characteristics distribution between those mothers who had successful ECV and those who had unsuccessful ECV (Table-1). In this study, $84 \%$ of those mothers who had successful ECV had spontaneous vertex vaginal delivery (Table-2), 
compared to only $9.3 \%$ in the unsuccessful ECV group, which is statistically significant finding( $\mathrm{P}$ value $=0.000$ ).

\section{Table-1}

Distribution of socio-demographic characteristics of study participants 
Variable

ECV Success status

Total P-value

Successful $n(\%) \quad$ Unsuccessful $n(\%)$

$n=109$

$n=43$

Age

$<20$

6(4.7)

3(6.9)

9(5.3)

0.397

20-25

33(30.8)

19(44.2)

52(34.1)

26-30

45(42.1)

16(37.2)

61(40.7)

31-35

17(15.9)

3(6.9)

20(13.3)

$>35$

$8(6.5)$

2(4.8)

10(7.2)

Weight of the mother $(\mathrm{kg})$

\begin{tabular}{lllll}
$\leq 65$ & $62(56.8)$ & $15(34.8)$ & $77(50.7)$ & 0.210 \\
\hline$>65$ & $47(43.2)$ & $28(65.2)$ & $75(49.3)$ &
\end{tabular}

\section{Religion}

\begin{tabular}{lllll} 
Orthodox & $58(53.3)$ & $27(62.8)$ & $85(56.0)$ & 0.410 \\
\hline Protestant & $19(17.8)$ & $8(18.6)$ & $27(17.7)$ & \\
\hline Muslim & $32(28.9)$ & $8(18.6)$ & $40(26.3)$ & \\
\hline
\end{tabular}

\section{Occupation}

\begin{tabular}{|c|c|c|c|c|}
\hline Unemployed & $11(9.3)$ & $2(4.6)$ & $13(8.0)$ & 0.426 \\
\hline Employed & 17(15.9) & 10(23.3) & $27(17.8)$ & \\
\hline Farmer & $5(4.7)$ & $1(2.3)$ & $6(4.0)$ & \\
\hline House wife & $72(66.4)$ & $30(69.8)$ & 102(67.3) & \\
\hline Other & $4(3.7)$ & - & $4(2.7)$ & \\
\hline \multicolumn{5}{|l|}{ Marital status } \\
\hline Married & 108(99.1) & 43(100.0) & 152(99.3) & 0.525 \\
\hline Divorced & $1(0.9)$ & - & $1(0.7$ & \\
\hline
\end{tabular}


Distribution of mode of delivery and fetal presentation at delivery

\begin{tabular}{|lllll|}
\hline Variable & ECV & & \multirow{2}{*}{$X^{2}$} & P-value \\
\cline { 2 - 4 } & Successful $n(\%)$ & Unsuccessful n(\%) & & \\
\hline Mode of delivery & & & & \\
\hline VD & $92(84.4)$ & $4(9.3)$ & 72.78 & 0.000 \\
\hline ABD & $3(2.8)$ & $14(32.6)$ & & \\
\hline CS & $14(12.8)$ & $25(58.1)$ & & \\
\hline
\end{tabular}

As shown in Table 3, the success rate of ECV for multiparous women was $73.6 \%(67 / 91, p$-value $=0.004)$ which was significantly higher than for nulliparous women who had a $68.8 \%(42 / 61)$ ECV success rate. For placental location, the highest success rate was obtained when the placenta was located in a posterior position $(85 \% ; 64 / 75$, $p$-value $=0.000 *)$. Among the types of breech, the highest success rate was achieved for a frank breech $(78.3 \% ; 74 / 94$, p-value $=0.02)$ as compared to non-frank breech $(39.7 \%$;

$35 / 58)$. Highest success rate was also achieved in those with women who thin abdominal wall $(78.8 \%, \mathrm{p}-$ value $=0.015)$, lateral fetal spine $(81.4 \%, p$-value $=0.028)$, and experienced no pain $(90.5 \%, p$-value $=0.001)$.

\section{Table 3}

Bivariate analysis of predictors of successful ECV 
Variable

ECV

$\operatorname{COR}(95 \% \mathrm{C} \mathrm{I})$

P-value

Successful n Unsuccessful n (\%)

(\%)

Maternal weight

$\begin{array}{lllll}\leq 65 & 64(79.5) & 15(20.5) & 1 & \\ >65 & 45(62.2) & 28(37.8) & 2.35(1.12-4.91) & 0.261\end{array}$

\section{Parity}

Nulliparous

42(68.8)

19(31.2)

1

Multiparous

67(73.6)

24(26.9)

1.02(0.39-1.67)

$0.004^{*}$

\section{Gestational age}

36-37 week

$30(65.2)$

16(34.8)

1

37-39 week

69(77.5)

20(22.5)

$0.49(0.23-1.02)$

0.059

>_40weeks

10(58.8)

7(41.2)

\section{AFV}

Low

Normal

High/

\section{Placentation}

Anterior

Lateral

Posterior

Descent of breech

Engaged

Unengaged

breech

Engaged

$$
8(70.0)
$$

95(70.4)

6(83.3)
24(44.4)

8(38.1)

11(14.7)
3(30.0)

39(29.5)

1(16.7)
1

0.97(0.24-3.98)

0.976

0.46(0.03-5.90)

0.556

30(58.3)

20(41.7)

1

79(77.5)

23(22.5)

0.41(0.19-0.85)

0.618

1.21(0.09-1.36)

0.000 *

\begin{tabular}{ll}
1 & \\
\hline $0.76(0.27-2.16)$ & 0.618 \\
\hline $1.21(0.09-1.36)$ & $0.000 *$
\end{tabular}


Type of breech

\begin{tabular}{lllll}
\hline Frank & $74(78.3)$ & $20(21.7)$ & $2.36(1.14-4.87)$ & $0.020^{*}$ \\
\hline Non frank & $35(60.3)$ & $23(39.7)$ & 1 & \\
\hline Amniotic fluid & & & & \\
\hline Low & $17(63.0)$ & $10(37.0)$ & 1 & 0.130 \\
\hline Normal & $92(90.3)$ & $10(9.7)$ & $0.28(0.10-0.76)$ & \\
\hline Fetal spine & & & & 0.463 \\
\hline Anterior & $49(75.4)$ & $16(24.6)$ & 1 & $0.028^{*}$ \\
\hline Posterior & $25(54.8)$ & $19(45.2)$ & $0.70(0.26-1.81)$ & $2.53(1.10-5.79)$ \\
\hline Lateral & $35(81.4)$ & $8(18.6)$ & & \\
\hline
\end{tabular}

Amniotic fluid

\begin{tabular}{lllll}
\hline Frank & $74(78.3)$ & $20(21.7)$ & $2.36(1.14-4.87)$ & $0.020 *$ \\
\hline Non frank & $35(60.3)$ & $23(39.7)$ & 1 & \\
\hline Amniotic fluid & & & & \\
\hline Low & $17(63.0)$ & $10(37.0)$ & 1 & 0.130 \\
\hline Normal & $92(90.3)$ & $10(9.7)$ & $0.28(0.10-0.76)$ & \\
\hline Fetal spine & & & 1 & 0.463 \\
\hline Anterior & $49(75.4)$ & $16(24.6)$ & $0.70(0.26-1.81)$ & $0.028^{*}$ \\
\hline Posterior & $25(54.8)$ & $19(45.2)$ & $2.53(1.10-5.79)$ & \\
\hline Lateral & $35(81.4)$ & $8(18.6)$ & & \\
\hline
\end{tabular}

\section{Abdominal wall}

Unremarkable
Thin
Fat
Level of pain

no pain

19(90.5) 2(9.5)

$18.04(3.48-93.6)$

$0.001 *$

Mild pain
Moderate pain
Sever pain
Uterine Tone

Medium

Soft

$75(81.5)$
$10(34.5)$
$5(37.5)$

$17(18.5)$
$19(65.5)$

$2.15(0.45-10.13)$

0.332

$15.83(2.05-80.06)$

$0.008 *$

$5(62.5)$

1

$16(48.5) \quad 17(51.5)$

83(92.2) $7(7.8)$

1
$2.23(0.76-6.53)$

$0.015^{\star}$

0.476 


\section{Number of}

attempt

\begin{tabular}{lllll}
\hline 1 & $15(65.0)$ & $7(35.0)$ & 1 & \\
\hline 2 & $66(83.5)$ & $13(16.5)$ & $0.36(0.12-1.09)$ & 0.072 \\
\hline 3 & $26(54.2)$ & $22(45.8)$ & $1.57(0.53-4.62)$ & 0.412
\end{tabular}

According to the results of multiple logistic regression analysis (Table-4), the variables that were significantly associated with the success of ECV were parity, thin abdominal wall, unengaged breech, placental location, pain level during procedure, and uterine tone. Multiparous women had a 1.4-times higher probability of success than nulliparous women $(95 \% \mathrm{Cl}, 0.07-2.35)$; thin abdominal wall had 3.5 fold increased success rate ( $95 \% \mathrm{Cl}, 0.29-42.40)$; unengaged breech had a 1.1 higher probably of success than engaged $(95 \% \mathrm{Cl}, 0.26-4.74)$; a posterior placental location increased the success rate by 1.14 times compared with an anterior placenta $(95 \% \mathrm{Cl}, 1.03-2.60)$; the odds of successful ECV was $14.68((95 \% \mathrm{Cl}, 1.65-34.97)$ when cases experienced no pain during the procedure; and tense uterine tone was significantly associated lower success rate as compared to soft tone (AOR= $0.08,95 \% \mathrm{Cl}, 0.016-$ 0.39). (Table 4).

\section{Table 4}

Multivariate logistic regression analysis of factors associated with success of ECV 


\section{Successful Unsuccessful n(\%)}

$n(\%)$

\section{Parity}

Nulliparous
Multiparous
Placentation

Anterior

30(55.6) 24(44.4)

1

Lateral

15(61.9) 8(38.1)

0.76(0.27-2.16)

1.12(0.20-6.29)

Posterior

64(85.3)

11(14.7)

1.21(0.09-1.36)

$1.14(1.03-2.60) \star$

\section{Descent of breech}

$\begin{array}{lll}\text { Engaged } & 30(58.3) & 20(41.7)\end{array}$

Unengaged

79(77.5) 23(22.5)

1

\section{Type of breech}

\begin{tabular}{lllll} 
Frank & $74(78.3)$ & $20(21.7)$ & $2.36(1.14-4.87)$ & $1.4(0.37-5.62)$ \\
\hline Non frank & $35(60.3)$ & $23(39.7)$ & 1 & \\
\hline
\end{tabular}

\section{Fetal spine}

\begin{tabular}{lllll}
\hline Anterior & $49(75.4)$ & $16(24.6)$ & 1 & \\
\hline Posterior & $25(54.8)$ & $19(45.2)$ & $0.70(0.26-1.81)$ & $0.63(0.13-3.06)$ \\
\hline Lateral & $35(81.4)$ & $8(18.6)$ & $2.53(1.10-5.79)$ & $1.32(0.34-5.19)$ \\
\hline Abdominal wall & & & & \\
\hline Unremarkable & $76(72.4)$ & $29(27.6)$ & 1 & \\
\hline Thin & $29(78.4)$ & $8(21.6)$ & $7.86(1.50-41.20)$ & $3.54(0.29-42.40)$ \\
\hline Thick & $4(25.0)$ & $6(75.0)$ & $0.72(0.29-1.76)$ & $0.28(0.05-1.38)$ \\
\hline
\end{tabular}

\section{Level of pain}

\begin{tabular}{lllll} 
no pain & $19(90.5)$ & $2(9.5)$ & $18.04(3.48-20.60)$ & $14.68(1.65-34.97)^{\star}$ \\
\hline Mild pain & $75(81.5)$ & $17(18.5)$ & $2.15(0.45-10.13)$ & $1.29(0.15-10.59)$
\end{tabular}


Moderate pain

Sever pain

\section{Uterine tone}

Medium

16(48.5)

83(92.2)

10(29.6)

Tense

soft

10(34.5)

19(65.5)

$15.83(2.05-30.06)$

$9.74(0.61-35.12)$

$5(37.5)$

$5(62.5)$

1

\section{Discussion}

In this study, the success rate of ECV was $71.7 \%$ and $84 \%$ of those mothers who had successful ECV had spontaneous vertex vaginal delivery. The variables that were significantly associated with the success of ECV were multiparty, thin abdominal wall, unengaged breech, posterior placenta, absence of pain during procedure, and soft uterine tone.

External cephalic version provides a means of reducing cesarean births, but an estimated $20-30 \%$ of eligible women are not being offered ECV $(10,11)$. The goal of ECV is to increase the proportion of vertex presentations among fetuses that were formerly in the breech position near term (12). According to the updated ACOG practice guidelines for ECV and based on a recent meta-analysis, the success rate of this procedure is reported to be $58 \%(13,14)$. Similarly, according to the SOGC guidelines, the procedure results in a cephalic presentation in approximately $60 \%$ of the time (15). The recent RCOG recommends that women undergoing ECV should be informed that the success rate of ECV is approximately $50 \%($ 16).

Overall, ECV is not widely practiced in East, Central, and Southern Africa, mainly owing to concerns related to safety and policy (17). Findings of our study have revolutionized this reality and one of the highest rates of success of ECV has been observed. From 152 ECV cases analyzed, 109(71.7\%) had successful ECV. This rate of ECV success in our study is higher than reports from different studies across most parts of the world. In a study done in Netherlands in 2015, among 2318 women having ECV procedure, 1093 (47.2\%) women had successful ECV, which is lower than the finding in our study (18). Similarly, one of the largest data on success of ECV, a cross-sectional analysis of 51,001 cases of ECVs in the USA (from 2010 to 2014 ) documented only a $58.5 \%$ success rate (19). Another large prospective data from UK, in which 2614 women who underwent ECV were analyzed, showed a $47 \%$ ECV success rate (95\% Cl $47.0-50.9 \%)(20)$, which is smaller than the finding in our study, and comparable to a finding of $45.3 \%$ success rate from a retrospective cohort study of 287 cases of ECV in Israel(21) and a Malaysian report of $51.4 \%$ success rate found in a recent retrospective review of 142 cases (22). A retrospective cohort study from Israel, done in 2019, in which $250 \mathrm{ECVs}$ were analyzed showed a success rate of $64.8 \%$ (23), which is again lower than that found in our study but higher than reports from other studies. Another recent study from Saudi Arabia reported a low success rate of $53.9 \%(24)$. Although there is scarcity of data on success of ECV in African settings, a $71.6 \%$ success rate found in our study is 
higher than findings from a prospective study done in Nigeria in which 111 ECV cases were studied and $74(66.7 \%)$ of these were successful (25).

A possible explanation for this unique finding of higher ECV success rate in our study, compared to the reports from the afro-mentioned studies from around the world, could be difference in patient body habitus. Although, obesity among reproductive age group in Ethiopia is variable, it is lower than that reported from developed world. For example, the prevalence of overweight and obesity among reproductive age group in Addis Ababa, the city where in this study was conducted, is only $20.6 \%$ (26). Majority(56.8\%) of the women who had successful ECV in our study had weight less than $65 \mathrm{KG}$, which indirectly corresponds to lower rate of obesity. Morbid obesity has been found consistently to be associated with low ECV success rate in different studies (27).

In the literature, a variety of factors have been mentioned as predictors of ECV success. Earlier, Newman et al. found that parity, estimated fetal weight, breech station and placental implantation site were the most useful predictors of ECV success (28). In the present study, multiparous women had a 1.4-times higher probability of success than nulliparous women $(95 \% \mathrm{Cl}, 0.07-2.35)$; thin abdominal wall had 3.5 fold increased success rate ( $95 \% \mathrm{Cl}, 0.29-42.40)$; unengaged breech had a 1.1 higher probably of success than engaged $(95 \% \mathrm{Cl}, 0.26-4.74)$; a posterior location of placenta increased the success rate by 1.14 times compared with an anterior placenta $(95 \% \mathrm{Cl}, 1.03-2.60)$; the odds of successful ECV was $14.68((95 \% \mathrm{Cl}, 1.65-34.97)$ when cases experienced no pain during the procedure; and tense uterine tone was significantly associated with lower success rate as compared to soft tone ( $\mathrm{AOR}=0.08,95 \% \mathrm{Cl}, 0.016$ $-0.39)$.

Most of the predictors of ECV success found in our study are consistent with reports from previous studies. For instance, P. A. Feyi-Waboso et al. found multiparty, flexed breech, unengaged breech, normal Amniotic fluid volume, and posterior placenta as favorable factors for successful ECV (25). Similarly, J. Burgos et al. from Spain found that the variables significantly associated with ECV success were parity, placental location, amount of amniotic fluid, and type of breech (29). Likewise, Melo et al and colleagues in their cohort study of 18 years' experience, demonstrated that multiparty, low uterine tone and high liquor volume were predictor s of ECV success (20). Another recent Canadian study demonstrated that non-engagement of the presenting part and an easily palpable fetal head were independently associated with success of ECV (30).

Women with a cephalic-presenting fetus at birth as a result of successful ECV are not at greater risk of obstetrical interventions at birth when compared with women with fetuses who spontaneously turn to a cephalic presentation in the third trimester (31). In this study, $84 \%$ of those mothers who had successful ECV had spontaneous vertex vaginal delivery, which is comparable to the finding of $85.7 \%$ vaginal delivery rate in a previous similar study from UK(20).

Strengths of this study include; prospective data and Sub-Saharan Africa study setting, where there is scarcity of data on experience and the finding of a higher ECV success rate. As discussed above, majority of ECV practice guidelines recommend to inform women that ECV has a success rate of $50-60 \%$. If 
substantiated with results from future studies, the finding of ECV success rate above this percentage in our study could challenge existing recommendations regarding ECV success rate and improve quality of ECV counselling for pregnant women complicated with breech presentation.

\section{Conclusion}

The success rate of ECV in this study is found to be $71.7 \%$, which is higher than reports from previous studies. Multiparty, absence of pain during procedure, posterior placenta, unengaged breech, soft uterine tone, and thin abdominal wall were significantly associated with ECV success.

\section{Abbreviations}

ACOG: American College of Obstetricians and Gynecologists

AOR: Absolute odds ration

ECV: External Cephalic version

Cl: Confidence interval

COR: Crude Odds Ratio

IRB: Institutional Review Board

KG: Kilogram

MFM : Maternal-Fetal-Medicine

RCOG: Royal College of Obstetricians and Gynecologists

SOGC: Society of obstetricians and Gynecologists Canada

SPHMMC: St.Pauls Hospital Millennium Medical College

\section{Declarations}

- Consent for publication

'Not applicable' 
Ethical clearance was obtained from St.Paul's Hospital Millennium Medical College IRB. Informed consent was obtained from each study subject participated in the study. All methods were performed in accordance with the relevant guidelines and regulations.

\section{- Availability of data and materials}

All data generated or analyzed during this study are included in this published article [and its supplementary information files].

\section{- Competing interests}

Authors have no financial nor non-financial competing interests

\section{- Funding}

This research didn't receive any funding for it's conduction or publication

\section{- Authors' contributions}

GM and TU developed the research concept and data collection process. AF and WG contributed data analysis, interpretation and manuscript write-up. All authors reviewed the manuscript.

\section{- Acknowledgements}

Authors would like to acknowledge St.Paul's Hospital Millennium Medical College

\section{References}

1. Hutton EK, Hannah ME, Barrett J. Use of external cephalic version for breech pregnancy and mode of delivery for breech and twin pregnancy: a survey of Canadian practitioners. J Obstet Gynaecol Can 2002;24:804-10

2. Goffinet F, Carayol M, Foidart JM, Alexander S, Uzan S, Subtil D, et al., for the PREMODA Study Group. Is planned vaginal delivery for breech presentation at term still an option? Results of an observational prospective survey in France and Belgium. Am J Obstet Gynecol 2006;194:1002-11.

3. Zhang J, Bowes WA, Fortney JA. Efficacy of external cephalic version: A review. Obstet Gynecol. 1993;82:306.

4. Hofmeyr GJ, Kulier R. External cephalic version for breech presentation at term. Cochrane Database Syst Rev 2005; Issue 1. Art. No.: CD000083. DOI: 10.1002/14651858.CD000083

5. Impey LWM, Hofmeyr GJ. External Cephalic Version and Reducing the Incidence of Breech Presentation. Green Top Guidelines No.20a. London: RCOG Press, 2006 
6. American College of Obstetrics and Gynecology (ACOG). Clinical management guidelines for obstetrician-gynecologists: External Cephalic Version. ACOG Practice Bull 2000;13:380-5. (reaffirmed 2009)

7. de Hundt M, Velzel J, de Groot CJ, Mol BW, Kok M. Mode of delivery after successful external cephalic version: a systematic review and meta-analysis. Obstet Gynecol. 2014 Jun;123(6):13271334. doi: 10.1097/AOG.0000000000000295. PMID: 24807332.

8. Hofmeyr GJ, Kulier R, West HM. External cephalic version for breech presentation at term. Cochrane Database Syst Rev. 2015 Apr 1;2015(4):CD000083. doi: 10.1002/14651858.CD000083.pub3. PMID: 25828903; PMCID: PMC6505738.

9. Onah, Hyacinth E., and Peter O. Nkwo. "External cephalic version: A survey of current practice among Nigerian obstetricians." Tropical Journal of Obstetrics and Gynaecology 21.1 (2004): 24-26

10. Vlemmix F, Rosman AN, te Hoven S, van de Berg S, Fleuren MA, Rijnders ME, et al. Implementation of external cephalic version in the Netherlands: a retrospective cohort study. Birth 2014;41:323-9

11. Caukwell S, Joels LA, Kyle PM, Mills MS. Women's attitudes towards management of breech presentation at term. J Obstet Gynaecol 2002;22:486-8

12. Mode of term singleton breech delivery. ACOG Committee Opinion No. 745. American College of Obstetricians and Gynecologists. Obstet Gynecol 2018;132:e60-3

13. External Cephalic Version: ACOG Practice Bulletin, Number 221. Obstet Gynecol. 2020 May;135(5):e203-e212. doi: 10.1097/AOG.0000000000003837. PMID: 32332415.

14. Grootscholten K, Kok M, Oei SG, Mol BW, van der Post JA. External cephalic version-related risks: a meta-analysis. Obstet Gynecol 2008;112:1143-51.

15. Krueger, Samantha, et al. "Labour Outcomes After Successful External Cephalic Version Compared With Spontaneous Cephalic Version." Journal of Obstetrics and Gynaecology Canada 40.1 (2018): 61-67

16. Impey LWM, Murphy DJ, Griffiths M, Penna LK on behalf of the Royal College of Obstetricians and Gynaecologists. External Cephalic Version and Reducing the Incidence of Term Breech Presentation. BJOG 2017; 124: e178-e192

17. Mukaindo AM, Wanyonyi SZ, Stones WR. External cephalic version in East, Central, and Southern Africa. Int J Gynaecol Obstet. 2012 Mar;116(3):228-31. doi: 10.1016/j.ijgo.2011.10.025. Epub 2011 Dec 22. PMID: 22196991

18. Beuckens A, Rijnders M, Verburgt-Doeleman GHM, Rijninks-van Driel GC, Thorpe J, Hutton EK. An observational study of the success and complications of 2546 external cephalic versions in low-risk pregnant women performed by trained midwives. BJOG 2016;123:415-423

19. Chaudhary, Shahrukh, Stephen Contag, and Ruofan Yao. "The impact of maternal body mass index on external cephalic version success." The Journal of Maternal-Fetal \& Neonatal Medicine 32.13 (2019): 2159-2165

20. Melo, P., et al. "External cephalic version at term: a cohort study of 18 years' experience." BJOG: An International Journal of Obstetrics \& Gynaecology 126.4 (2019): 493-499 
21. Salzer, Liat, et al. "Predictors of successful external cephalic version and assessment of success for vaginal delivery." The Journal of Maternal-Fetal \& Neonatal Medicine 28.1 (2015): 49-54.

22. Lim, Pei Shan, et al. "Successful external cephalic version: factors predicting vaginal birth." The Scientific World Journal 2014 (2014).

23. Isakov, Ofer, et al. "Prediction of success in external cephalic version for breech presentation at term." Obstetrics \& Gynecology 133.5 (2019): 857-866

24. Hussin, O. A., M. A. Mahmoud, and M. M. Abdel Fattah. "External cephalic version for breech presentation at term: predictors of success, and impact on the rate of caesarean section." EMHJEastern Mediterranean Health Journal, 19 (2), 162-166, 2013 (2013)

25. Feyi-Waboso PA, Selo-Ojeme CO, Selo-Ojeme DO. External cephalic version (ECV): experience in a sub-Saharan African hospital. J Obstet Gynaecol. 2006 May;26(4):317 - 20. doi: 10.1080/01443610600594948. PMID: 16753680

26. Tebekaw $Y$, Teller $C$, Colón-Ramos $U$. The burden of underweight and overweight among women in Addis Ababa, Ethiopia. BMC Public Health 2014;14:1-11. 10.1186/1471-2458-14-1126 [PMC free article] [PubMed] [CrossRef] [Google Scholar]

27. Chaudhary S, Contag S, Yao R. The impact of maternal body mass index on external cephalic version success. J Matern Fetal Neonatal Med. 2019 Jul;32(13):2159-2165. doi:

10.1080/14767058.2018.1427721. Epub 2018 Jan 21. PMID: 29355061)

28. Newman RB et al. Predicting success of external cephalic version. American Journal of Obstetrics and Gynecology, 1993, 169:245-249

29. Burgos J, Melchor JC, Pijoán JI, Cobos P, Fernández-Llebrez L, Martínez-Astorquiza T. A prospective study of the factors associated with the success rate of external cephalic version for breech presentation at term. Int J Gynaecol Obstet. 2011 Jan;112(1):48-51. doi:

10.1016/j.ijgo.2010.07.023. Epub 2010 Sep 25. PMID: 20870233

30. Hutton, Eileen K., et al. "Predictors of success of external cephalic version and cephalic presentation at birth among 1253 women with non-cephalic presentation using logistic regression and classification tree analyses." Acta obstetricia et gynecologica Scandinavica 96.8 (2017): 1012-1020.

31. Krueger, Samantha, et al. "Labour Outcomes After Successful External Cephalic Version Compared With Spontaneous Cephalic Version." Journal of Obstetrics and Gynaecology Canada 40.1 (2018): $61-67$ 